\title{
Clinical Application Analysis of Painless Gastrointestinal Endoscopy in Gastroenterology
}

\author{
Ling Zhang \\ Luohe Medical College, Luohe, Henan, 462000
}

Keywords: gastroenterology; painless gastrointestinal endoscopy; examination

\begin{abstract}
Objective: To observe the application effect of painless gastrointestinal endoscopy in the treatment of digestive diseases and provide reference for clinical application. Methods: A total of 144 patients who underwent gastrointestinal endoscopy in the Department of Gastroenterology were randomly divided into two groups, 72 in each group. Patients in the control group underwent routine gastrointestinal endoscopy. The patients in the observation group underwent painless gastrointestinal endoscopy. The operation time, examination results, pain and adverse reactions of the two groups were compared. Results: The physiological indexes (heart rate, blood pressure, etc.) of the observation group were lower than those of the control group before and after the examination. The observation group had short operation time, mild pain and low adverse reaction rate. The difference was statistically significant $(\mathrm{P}<0.05)$. Conclusion: Painless gastrointestinal endoscopy has the characteristics of small trauma, mild pain, low adverse reaction rate and good compliance. It can be used as an ideal method for gastrointestinal examination of gastroenterology.
\end{abstract}

\section{Introduction}

In recent years, common gastrointestinal endoscopy and painless gastrointestinal endoscopy have been used clinically to treat diseases related to gastroenterology. In the diagnosis and treatment of common gastroscope, there are many unpleasant stimuli, which bring different degrees of discomfort to the patients. Most patients will refuse to accept the diagnosis and treatment of common gastroscope because of fear of pain, thus delaying the best treatment timing of digestive diseases. treatment effect. Painless gastrointestinal endoscopy is the first choice for the diagnosis and treatment of digestive diseases. It has the advantages of operability, safety and high efficiency, and is widely used in hospital digestive medicine. In order to alleviate the discomfort during the diagnosis and treatment of patients with gastrointestinal endoscopy and improve patient satisfaction, in recent years, our hospital has widely used painless gastrointestinal microscopy to treat digestive diseases, and achieved good results. The summary report is as follows.

\section{Materials and Methods}

Clinical data: From February 2014 to February 2016, 144 patients who underwent gastrointestinal endoscopy in the Department of Gastroenterology, First Affiliated Hospital of Luohe Medical College, were selected as the study subjects, including 67 males and 77 females, aged 6-75. At the age of the patients, patients were randomly divided into a control group and a control group. There were 72 patients in the control group, including 33 males and 39 females; aged 20-73 years, mean $(45.73 \pm 11.55)$ years old; body weight $45-81 \mathrm{~kg}$, mean $(64.53 \pm 7.35) \mathrm{kg}$. There were 72 patients in the observation group, 27 males and 45 females; aged 21-76 years old, average $(44.58 \pm 10.24)$ years old; weight $47-75 \mathrm{~kg}$, average $(63.75 \pm 7.22) \mathrm{kg}$. There were no significant differences in gender, age and weight between the two groups $(\mathrm{P}>0.05)$, which were comparable.

Preoperative preparation The patient does not smoke 1 day before the test to avoid intubation due to cough during the examination. Carefully ask the patient whether they have undergone endoscopy, whether there are complications (such as high blood pressure, heart disease, diabetes); do an electrocardiogram, laboratory-related examinations; patients with sputum fast before surgery, if 
necessary, enema, do Good gastrointestinal preparation; prepare the instruments and drugs necessary for gastrointestinal endoscopy, and sterilize and disinfect the instruments; establish venous access and remove the dentures. Provide adequate oxygen to the patient while monitoring the patient for ECG.

Method of operation Control group: Patients underwent routine gastrointestinal endoscopy. 10 minutes before the endoscopy, the patient took about $2 \mathrm{~mL}$ of $2 \%$ lidocaine cement and waited for the pharynx to enter the mirror. Before the colonoscopy, apply $2 \%$ lidocaine glue to the anus position and the front end of the lens. Mirror inspection. Observation group: The patient underwent painless gastrointestinal endoscopy. Oxygen was inhaled through the nasal cannula, intravenous fentanyl $(0.5 \mu \mathrm{g} / \mathrm{kg})$, midazolam $(1 \mathrm{mg})$, and slow intravenous injection of propofol $(50 \mathrm{mg} / \mathrm{min})$ for $3 \mathrm{~min}$. The eyelash reflex disappears and the administration is stopped, and the examination operation is performed according to the requirements of the gastrointestinal endoscopy. During the examination, the propofol dose is added according to the actual situation of the patient.

Observation indicators Monitoring and comparing the heart rate, oxygen saturation and blood pressure during the operation of the two groups of patients; Observing the patient's adverse reactions and assessing the patient's compliance and pain.

Evaluation of pain level: Mullertr score and visual analog scale (VAS) method were used as the standard of patient self-feeling and physician judgment. Rating: 0 points, no pain; 1 point, mild pain, does not affect activity; 2 points, moderate pain, activity can continue; 3 points, strong pain, affecting activity; 4 points, strong pain, Unable to continue

Work. Compliance evaluation: excellent patient response did not see adverse reactions, good compliance; good for patients with mild discomfort during the operation, general compliance; poor operation in patients with strong discomfort, poor compliance.

Statistical processing using SPSS 14. 0 Statistical software for data analysis, the measurement data is expressed as $\mathrm{x} \pm \mathrm{s}$, the t test is used for comparison between the two groups, the chi-square test is used for the comparison of the count data, and the rank sum test is used for the comparison of the grade data, with $\mathrm{P}<0.05$ as the difference. Statistical significance.

\section{Results}

Before and after the examination of heart rate, systolic blood pressure, diastolic blood pressure and oxygen saturation before and after the examination, there was no significant difference in heart rate, systolic blood pressure, diastolic blood pressure and oxygen saturation between the two groups $(\mathrm{P}>0.05)$. After the examination, the above indicators of the observation group were superior to the control group, and the difference was statistically significant $(\mathrm{P}<0.05)$.

The pain scores and adverse reactions of the two groups were lower than those of the control group. The observation time of the observation group was shorter than that of the control group, and the adverse reactions were less than the control group. The difference was statistically significant $(\mathrm{P}<0.05)$.

\section{Discussion}

Gastrointestinal diseases are most common with gastrointestinal diseases and are closely related to patients' lifestyles and eating habits. With the rapid development of the economy, people's life rhythm has accelerated. In order to adapt to the fast-paced life, most people have developed bad habits, digestive diseases have increased, and the incidence of gastrointestinal diseases has increased year by year. Clinically, the diagnosis and treatment of gastroenterology is more and more common with gastrointestinal endoscopy. When performing a gastroscopy, the patient usually takes the left lateral position, the head is slightly forward, and contains the chest. Untie the collar to avoid affecting the neck activity. Guide the black plastic wrapped with an endoscope and a diameter of about $1 \mathrm{~cm}$. The slender tube of the optical fiber is inserted into the esophagus $\rightarrow$ stomach $\rightarrow$ duodenum through the patient's mouth, and the light emitted by the light source can be deflected by the light guiding fiber, and the doctor can observe the condition of different parts of the upper 
digestive tract of the patient. According to the needs of diagnosis and treatment, the gastroscope can be inserted into the clip for biopsy. Colonoscopy: The colonoscopy was inserted into the ileum and cecum along the intestine of the patient's anus, and the colon was observed from the mucosal side. Colonoscopy can effectively observe changes in the colonic mucosa and help to diagnose large intestinal mucosal diseases. In the clinical application process, gastrointestinal endoscopy is divided into three steps: intestinal cleansing, gastrointestinal endoscopy and information diagnosis. With the continuous advancement of medical technology, painless gastrointestinal microscopy technology has gradually replaced traditional gastrointestinal mirror technology, allowing patients to be treated under anesthesia, and the scope of indications for painless gastrointestinal endoscopy has been further expanded (such as hypertension, Cardiovascular and cerebrovascular diseases, etc., while effectively reducing the pain of patients and improving the therapeutic effect.

Gastrointestinal examination is an interventional treatment. Patients with conventional gastrointestinal endoscopy are prone to various types of discomfort, such as cough and vomiting caused by pharyngeal discomfort, severe nausea, abdominal pain, vomiting caused by gastrointestinal tube traction, etc., severe pain can even cause serious complications such as sudden cardiac arrest. Many patients choose to give up medical treatment because they can't stand the discomfort, check the poor compliance, delay the diagnosis, and lose the best treatment opportunity. The painless gastrointestinal endoscopy is based on the routine gastrointestinal endoscopy combined with an appropriate amount of anesthetic to assist the operation. The anesthetic used has the advantages of quick effect and short efficacy, and allows the patient to undergo examination and treatment in a state where consciousness is temporarily disappeared. In order to avoid examination of various adverse reactions produced during treatment.

During the operation, the patient does not feel any pain. After the examination, the patient can recover consciousness within 1 to 11 minutes. After waking, the whole process is generally not remembered. At present, anesthetics such as propofol and zodiazepine are usually used for painless gastrointestinal endoscopy at home and abroad. These drugs can cause patients to form anterograde amnesia and no implicit memory, so the patient seems to know the examination during the examination. However, after the recovery of consciousness, it is impossible to recall the inspection process. Propofol is an intravenous anesthetic with a short duration of action, effective onset, and rapid recovery. The dose is proportional to the inhibitory effect of consciousness. The patient usually has an intravenous injection of 0 . Loss of consciousness $(1 \sim 2 \mathrm{mg} / \mathrm{kg})$ after $5 \sim 1 \mathrm{~min}$, no adverse reactions after waking. However, the drug has certain toxic side effects, such as inhibiting the myocardium, dilating the peripheral blood vessels, etc., and lowering the heart rate can lower the blood pressure of the patient, so the injection speed should be strictly controlled, and the injection should be as short as possible. Clinical practice shows that patients often have blood pressure, heart rate and other obvious changes before and during routine gastrointestinal endoscopy. If the patient has high blood pressure or cardiovascular and cerebrovascular diseases, the examination may induce disease and endanger the patient. Life is healthy. By injecting a sedative into a patient, the patient's tolerance and self-consciousness can be effectively improved, and the difficulty of diagnosis and treatment can be reduced. However, before the painless gastrointestinal endoscopic surgery, the patient's biochemical indicators should be carefully observed. The patient can take oxygen and keep the breath open during the examination. Monitor the patient's blood pressure, heart rate and oxygen saturation to avoid respiratory depression. Waiting for the situation. By administering a sedative to the patient for painless gastrointestinal endoscopy, the difficulty of diagnosis and treatment of gastrointestinal endoscopy can be alleviated; the patient is in an anesthetic state and is more favorable for biopsy, which can effectively improve the success rate of pathological materials and the accuracy of subsequent diagnosis.

The observation results showed that the physiological indexes such as heart rate, systolic blood pressure, diastolic blood pressure and blood oxygen saturation of the observation group were small, which was significantly better than the control group, and no obvious adverse reactions occurred. After the examination, the patient was awake within 3 to 10 minutes without communication problems. Patients in the observation group had better compliance, which was convenient for 
doctors to perform operation check, identify lesions, shorten operation time, make gastrointestinal endoscopy diagnosis easier, and patients are more acceptable.

In summary, the painless gastrointestinal endoscopy technique is used for the diagnosis and treatment of medical diseases. The patient has small fluctuations in physiological indexes, good clinical efficacy, mild adverse reactions, less complications, and high safety. It can be widely used in large intestinal mucosal diseases and digestive tracts. Diagnosis and treatment of diseases such as bleeding.

\section{References}

[1] Wu Yongyong. Clinical application of painless gastrointestinal endoscopy in gastroenterology [J]. Modern Diagnosis and Treatment, 2013, 9(10): 2277-2278.

[2] Peng Hemei, Xiao Xiang. Routine and painless gastrointestinal endoscopy safety study [J]. Chinese Contemporary Medicine, 2012, 19(16): 91-92.

[3] Huang Lihua. Clinical application of painless gastrointestinal endoscopy in gastroenterology [J]. Contemporary Medicine, 2012, 18(25): 51 